\title{
KEYSTREAM SEQUENCES WITH A GOOD LINEAR COMPLEXITY PROFILE FOR EVERY STARTING POINT
}

\author{
Harald Niederreicer \\ Mathematical Institute, Austrian Academy of Sciences \\ Dr.-Ignaz-Seipel-Platz 2 \\ A-1010 Vienna, Austria
}

\section{INTRODUCTION AND PROBABILISTIC RESULTS}

The linear complexicy profile was introduced by Rueppel [7], [8, Ch. 4] as a cool for the assessment of keystream sequences with respect to randomness and unpredictability properties. In the following let $\boldsymbol{F}$ be an arbitrary field. We recall that a sequence of elements of $F$ is called a kth-order linear feedback shift reglster (LFSR) sequence if it satisfies a kth-order linear recursion with constant coefficients from F. The zero sequence $0,0, \ldots$ is viewed as an LFSR sequence of order 0 . Now let $S$ be an arbitrary sequence $s_{1}, s_{2}, \ldots$ of elements of $F$. For a positive integer $n$ the (local) linear complexity $L_{n}(S)$ is defined as the least $k$ such that $s_{1}, s_{2}, \ldots, s_{n}$ form the first $n$ terms of a kth-order LFSR sequence. The sequence $\mathrm{L}_{1}(\mathrm{~S}), \mathrm{L}_{2}(\mathrm{~S}), \ldots$ of integers is called the linear complexiry profile (LCP) of $S$. For bastc facts about the LCP see [4], [7], [8, Ch. 4].

The study of the LCP leads to the requirement that the LCP of a keystream sequence should simulate the LCP of a random sequence. Typical features of the LCP of a randon sequence were investigated in [5], [7], [8, Ch. 4]. An additional requiremenc was pointed out by Piper [6], namely that a keystream sequence should have an acceptable LCP for every starting point. In other words, if $S_{m}$ is the shifted sequence $s_{m+1}, s_{m+2}, \ldots$, then $s_{m}$ should have an acceptable LCP for every $m=0,1, \ldots$. In the present paper we are mainly concerned with this requirement.

A basic technical device in this work is the identification of the sequence $S$ with its generating function $\sum_{i=1}^{\infty} s_{i} x^{-i}$, viewed as an element of the field $G=F\left(\left(x^{-1}\right)\right)$ of formal Laurent series in $x^{-1}$ over $F$ (compare also with [4]). For the probabilistic theory we take $F$ to be the Finite field $F$ with $q$ elements, where $q$ is an arbitrary prime power. Note that in practical stream cipher applications we have the binary case $q=2$. The uniform probabilicy measure on $F_{q}$ assigns 
the measure $1 / \mathrm{q}$ to each element of $\mathrm{F}_{\mathrm{q}}$. This probability measure induces the complete product probability measure $h$ on the set $F \underset{q}{\infty}$ of all sequences of elements of $F_{q}$ by a standard procedure of probability theory (see [2, Sec. 4]). It is easily seen that the measure $h$ is the same as the Haar measure used in [5] when the latter measure is transferred from the set of all generating functions to the set $F_{q}^{\infty}$. We say that a stated property of elements of $F_{q}^{\infty}$ holds h-almost everywhere (h-a.e.) if the property holds for all elements of a subset of $F_{q}^{\infty}$ of h-measure 1 . A property that holds h-a.e. can be viewed as a typical property of a random sequence of elements of $\xi_{q}$.

Theorem 1. Let $\pi$ be a property of elements of $F_{q}^{\infty}$ that holds h-a.e. Then the set of all $s \in F_{q}^{\infty}$ for which $\Pi$ holds simultaneously for all shifted sequences $S_{m}$, $\mathrm{m}=0,1, \ldots$, has $\mathrm{h}$-measure 1 .

Proof. Let $P$ be the set of all elements of $F_{q}^{\infty}$ which have the property $\Pi$ and let $V$ be the set of all $s \in F_{q}^{\infty}$ for which $s_{m} \in P$ for all $m=0,1, \ldots$. Let $\tau$ be the unilateral shift operator on $\mathrm{F}_{\mathrm{q}}^{\infty}$ defined by

$$
\tau\left(s_{1}, s_{2}, \ldots\right)=\left(s_{2}, s_{3}, \ldots\right) \quad \text { for } \quad\left(s_{1}, s_{2}, \ldots\right) \in F_{q}^{\infty} \text {. }
$$

Then $s_{m}=\tau^{m} s$, thus $s_{m} \in P$ if and only if $S$ lies in the mth iterated inverse image $\tau^{-m_{P}}$. Therefore $V=\bigcap_{m=0}^{\infty} \tau^{-m_{P}}$. Now $\tau$ is measure-preserving with respect to $h$ by $[1, \mathrm{Ch} .1]$, and so $h\left(\tau^{-m_{P}}\right)=h(P)=1$ for all $\mathrm{m}$. Thus we get $h(V)=1$.

Theorem 2. If $F=F_{q}$, then h-a.e. we have

$$
\begin{aligned}
& \lim _{n \rightarrow \infty} \frac{L_{n}\left(S_{m}\right)}{n}=\frac{1}{2} \quad \text { for } m=0,1, \ldots, \\
& \lim _{n \rightarrow \infty} \sup _{n \rightarrow \infty} \frac{L_{n}\left(s_{m}\right)-(n / 2)}{\log n}= \pm \frac{1}{2 \log q} \text { for } m=0,1, \ldots .
\end{aligned}
$$

Proof. This follows from Theorem 1 and $[5$, Theorems 7 and 10]. 0

From Theorem 1 one can deduce various other probabilistic results. For example, all the probabilistic results in [5] hold simultaneously for.all shifted sequences $s$, with Theorem 2 just covering two instances that are easy to state. In particular, the results about the distribution of partial quotients in the continued fraction expansion of a random generating function $S$ hold simultaneously for all $S_{m}$. In a nutshe11, Theorem 1 provides the basis for saying that Piper's requirement is met by random sequences of elements of $F_{q}$. 


\section{SEQUENCES WITH A GOOD LINEAR COMPLEXITY PROFILE}

We introduce a class of sequences for which the LCP is close to that of a random sequence and for which this property is retained under shifts. Put Log $t=\max (1$, $\log t)$ for $t \geqslant 1$.

Definition 1. A sequence $S$ of elements of the field $F$ has a good LCP if there exists a constant $C$ (which may depend on $S$ ) such that

$$
\left|L_{n}(S)-\frac{n}{2}\right| \leq C \log n \quad \text { for } n=1,2, \ldots
$$

If $F=F_{q}$, then it follows from [5, Theorem 10] that the property of having a good LCP holds h-a.e. For further analysis we need the connection between the LCP and continued fractions as developed in [4]. Every $S \in G$ has a unique continued fraction expansion

$$
S=A_{0}+1 /\left(A_{1}+1 /\left(A_{2}+\ldots\right)\right)=:\left[A_{0}, A_{1}, A_{2}, \ldots\right]
$$

with $A_{j} \in F[x]$ for $j \geqslant 0$ and $\operatorname{deg}\left(A_{j}\right) \geqslant 1$ for $j \geqslant 1$. This expansion is finite for rational $S$ and infinite for irrational. $S$. The polynomials $A_{j}, j \geqslant 1$, are called the partial quotients and $A_{0}=:$ Pol(s) is the polynomial part of $S$. The polynomials $P_{j}$ and $Q_{j}$ are defined as in [4] and $P_{j} / Q_{j}$ is called a convergent. We note that

$$
\operatorname{deg}\left(Q_{j}\right)=\sum_{i=1}^{j} \operatorname{deg}\left(A_{i}\right) \text { for } j \geq 1 \text {. }
$$

Then we have the following formula [4, Theorem 1].

Lemma 1. For any sequence $S$ of elements of $F$ and any $n \geqslant 1$ we have $L_{n}(S)=$ $\operatorname{deg}\left(Q_{j}\right)$, where $j \geqslant 0$ is uniquely determined by the condition

$$
\operatorname{deg}\left(Q_{j-1}\right)+\operatorname{deg}\left(Q_{j}\right) \leq n<\operatorname{deg}\left(Q_{j}\right)+\operatorname{deg}\left(Q_{j+1}\right)
$$

Proposition 1. If the generating function of a sequence $S$ is irrational and $\operatorname{deg}\left(A_{j}\right) \leqslant C \log j$ for all $j \geqslant 1$, where $C$ is a constant, then

$$
\left|L_{n}(S)-\frac{n}{2}\right| \leq \frac{C}{2} \text { Log } n \quad \text { for a } 11 \quad n \geqslant 1
$$


Proof. Consider an $n$ satisfying (2) and first assume $j \geqslant 2$. Then $L_{n}(S)=\operatorname{deg}\left(Q_{f}\right)$ by Lemma 1 , hence using ( 1 ) we- get

$$
\begin{aligned}
& \left|L_{n}(S)-\frac{n}{2}\right|=\left|\operatorname{deg}\left(Q_{j}\right)-\frac{n}{2}\right| \leq \frac{1}{2} \max \left(\operatorname{deg}\left(A_{j}\right), \operatorname{deg}\left(A_{j+1}\right)\right) \\
& \leq \frac{C}{2} \log (j+1) \leq \frac{C}{2} \log \left(\operatorname{deg}\left(Q_{j}\right)+1\right) \leq \frac{C}{2} \log n .
\end{aligned}
$$

The proposition is easily checked for $j=0$ and $j=1$.

Proposition 2. If a sequence $S$ satisfies $\left|L_{n}(S)-(n / 2)\right| \leqslant C$ Log $n$ for some constant $C \geq 1$ and all $n \geq 1$, then its generating function is irrational and

$$
\operatorname{deg}\left(A_{j}\right)<(4 C \log C+8 C) \log j \quad \text { for all } j \geq 1
$$

Proof. The given condition on $L_{n}(S)$ implies $\lim _{n \rightarrow \infty} L_{n}(S)=\infty$, and so the generating function of $S$ is irrational. To prove (3) we $\overrightarrow{p r o c e d}^{\infty}$ by contradiction, and we let $j$ be the least index such that the inequality in (3) does not hold. First assume $j=1$, so that

$$
\operatorname{deg}\left(A_{1}\right) \geqslant 4 C \log C+8 C .
$$

For $n=\operatorname{deg}\left(A_{1}\right)-1$ we have $n \geq 4 C \log C+7 C$ and also $L_{n}(S)=0$ by Lemma 1 . Thus

$$
\left|L_{n}(S)-\frac{n}{2}\right|=\frac{n}{2} \geqslant \frac{4 C \log C+7 C}{2 \log (4 C \log C+7 C)} \log n
$$

since the function $t / \log t$ is increasing for $t \geqslant e$. By distinguishing between the cases $1 \leqslant \mathrm{C} \leqslant \mathrm{e}$ and $\mathrm{C}>\mathrm{e}$ one shows that

$$
4 \log C+7>2 \log (4 C \log C+7 C) \text { for all } C \geqslant 1 \text {, }
$$

and so $\left|L_{n}(S)-(n / 2)\right|>C$ Log $n$, a contradiction. Now let $j \geqslant 2$. Then with $c_{1}=$ $4 C \log C+8 C$ we have $\operatorname{deg}\left(A_{i}\right)<C_{1} \log i$ for $1 \leqslant i<j$. Together with (1) we get

$$
\log \operatorname{deg}\left(Q_{j}\right)<\log \left(\operatorname{deg}\left(A_{j}\right)+C_{1} \sum_{i=1}^{j-1} \log i\right) \leq \log \left(\operatorname{deg}\left(A_{j}\right)+C_{1}(j-1) \log j\right) .
$$

Since the function $t^{-1} \log \left(t+C_{1}(j-1) \log j\right)$ is decreasing for $t \geqslant e$ and since $\operatorname{deg}\left(A_{j}\right) \geqslant c_{1} \log j$, it follows that

$$
\log \operatorname{deg}\left(Q_{j}\right)<\frac{\log \left(C_{1} j \log j\right)}{C_{1} \log j} \operatorname{deg}\left(A_{j}\right) \text {. }
$$

For $n=\operatorname{deg}\left(Q_{j-1}\right)+\operatorname{deg}\left(Q_{j}\right)$ we have by Lemma 1 and $(1)$, 


$$
\begin{aligned}
& \left|L_{n}(S)-\frac{n}{2}\right|=\left|\operatorname{deg}\left(Q_{j}\right)-\frac{1}{2}\left(\operatorname{deg}\left(Q_{j-1}\right)+\operatorname{deg}\left(Q_{j}\right)\right)\right|=\frac{1}{2} \operatorname{deg}\left(A_{j}\right) \\
& >\frac{C_{1} \log j}{2 \log \left(C_{1} j \log j\right)} \log \operatorname{deg}\left(Q_{j}\right)>\frac{C_{1} \log j}{2 \log \left(C_{1} j \log j\right)} \log \frac{\pi}{2} .
\end{aligned}
$$

Now $n \geqslant \operatorname{deg}\left(A_{j}\right)+2 \operatorname{deg}\left(A_{j-1}\right) \geqslant c_{1} \log j+2$, and so

$$
\left|L_{n}(S)-\frac{\pi}{2}\right|>\frac{C_{1} \log j}{2 \log \left(C_{1} j \log j\right)}\left(1-\frac{\log 2}{\log \left(C_{1} \log j+2\right)}\right) \log n \text {. }
$$

Therefore, to arrive at the contradiction $\left|L_{n}(S)-(n / 2)\right|>C \log n$, it suffices to show that

$$
(2 \log C+4)\left(1-\frac{\log 2}{\log \left(C_{1} \log j+2\right)}\right) \geq \frac{\log \left(C_{1} j \log j\right)}{\log j} .
$$

To prove (4), we first consider $j=2$. Then (4) attains the simpler form

$$
(2 \log C+4)\left(1-\frac{\log 2}{\log \left(C_{1}+2\right)}\right) \geqslant \log \left(2 C_{1}\right) .
$$

We have

$$
(2 \log C+4)\left(1-\frac{\log 2}{\log \left(C_{1}+2\right)}\right) \geqslant(2 \log C+4)\left(1-\frac{\log 2}{\log 14}\right)>(1.47) \log C+2.94 .
$$

For $1 \leq c \leq e$ it follows that

$$
(2 \log C+4)\left(1-\frac{\log 2}{\log \left(C_{1}+2\right)}\right)>4.41>\log (24 e) \geqslant \log (24 C)=\log \left(2 C_{1}\right) .
$$

Since the function $(0.47) t-\log (t+2)$ is increasing for $t \geqslant 1$, we obtain $(0.47) t+$ $0.86>\log (t+2)$ for $t \geq 1$, and so

$$
(0.47) \log c+0.86>\log (\log c+2) \text { for } c \geq e \text {. }
$$

It follows that

$$
(1.47) \log C+2.94>\log C+\log 8+\log (\log C+2)=\log \left(2 C_{1}\right) \text { for } c \geqslant e \text {, }
$$

hence (4) is shown for $j=2$. For $j \geq 3$ we have

$$
1-\frac{\log 2}{\log \left(C_{1} \log j+2\right)} \geq 1-\frac{\log 2}{\log (12 \log 3+2)}>0.74
$$

Since the function $\log \left(C_{1} t \log t\right) / \log t$ is decreasing for $t \geq e$, we obtain (4) for $j \geq 3$ if we can show that 


$$
(1.48) \log C+2.96 \geq \frac{\log \left(3 C_{1} \log 3\right)}{\log 3}
$$

For $1 \leqslant \mathcal{C} \leqslant$ e we have

$$
(1.48) \log C+2.96=4.44>\frac{\log (36 e \log 3)}{\log 3} \geq \frac{\log \left(3 C_{1} \log 3\right)}{\log 3},
$$

hence (5) holds. The function $(0.62) t-\log (t+2)$ is increasing for $t \geq 1$, thus $(0.62) t+0.67>\log (t+2)$ for $t \geq 1$, and so

$$
(0.62) \log c+0.67>\log (\log C+2) \text { for } c \geq e \text {. }
$$

Adding $\log C+\log (12 \log 3)$ on both sides and then dividing by $\log 3$, we obtain (5).

Theorem 3. A sequence $S$ has a good LCP if and only if its generating function is irrational and there exists a constant $C$ (which may depend on $S$ ) such that $\operatorname{deg}\left(A_{j}\right)$ $\leq \mathrm{C}$ Log $j$ for all $\mathrm{j} \geq 1$.

Proof. This follows from Propositions 1 and 2 .

Note that the generating function of $S$ is irrational if and only if $S$ is not an LFSR sequence (see [4, Sec. 2]). We now use the valuation $v$ on $G$ introduced in [4, Sec. 3]. We also write $\operatorname{Fr}(S)=S-\operatorname{Pol}(S)$ for $S \in G$.

Lemma 2. If $S \in G$ and $f, g \in F[x]$ with $f \neq 0$ and $v(E S-g)<-v(f)$, then $f=D Q_{j}$ and $g=D P$ for some $j \geqslant 0$, where $D \in F[x]$ and $D \neq 0$.

Proof. We have $v(f S-g)<0$, so [4, Lemma 3] can be applied and yields

$f=\sum_{k=0}^{j} D_{k} Q_{k}, g=\sum_{k=0}^{j} D_{k} P_{k}$, where $D_{k} \in \mathbb{F}[x], v\left(D_{k}\right)<v\left(A_{k+1}\right)$ for $0 \leq k \leq j$, and $D_{j} \neq 0$; moreover, if $i$ is the least index with $D_{i} \neq 0$, then $v(E S-g)=$ $v\left(D_{i}\right)-v\left(Q_{i+1}\right)$. If we had $i<j$, then

$$
v(E S-g) \geq v\left(D_{i}\right)-v\left(Q_{j}\right) \geq-v\left(Q_{j}\right) \geq-v(f)
$$

a contradiction. Thus $i=j$, hence $f=D Q_{j}$ and $g=D P_{j}$ with $D=D_{j} \cdot \square$

Lemma 3. Let $s \in G$ and $f, g \in F[x]$ with $f_{g} \neq 0$. Let $\frac{f}{g} S=\left[A_{0}^{1}, A_{1}^{\prime}, A_{2}^{1}, \ldots\right]$ and let $P_{j} / Q_{j}^{\prime}$ be the corresponding convergents. If $v\left(A_{j}^{\prime}\right)>v(f)+v(g)$ for some $j \geq 1$, then there exists an $i \geq 1$ such that $v\left(A_{j}^{\prime}\right) \leq v\left(A_{i}\right)+v(E)+v(g)$ and $v\left(Q_{i-1}\right) \leqslant$ $v\left(Q_{j-1}^{\prime}\right)+v(f)$. 
Proof. By $[4$, eq. $(6)]$ we have $v\left(Q_{j-1}^{\prime} \frac{f}{g} S-P_{j-1}^{\prime}\right)=-v\left(Q_{j-1}^{\prime}\right)-v\left(A_{j}^{\prime}\right)$, hence $v\left(A_{j}^{\prime}\right)>v(f)+v(g)$ implies $v\left(f Q_{j-1}^{\prime} S-g P_{j-1}^{\prime}\right)<-v\left(f Q_{j-1}^{\prime}\right)$. Then Lemna 2 yields $E Q_{j-1}^{\prime}=D Q_{i-1}$ and $g P_{j-1}^{\prime}=D P_{i-1}$ for some $i \geq 1$, where $D \in F[x]$ and $D \neq 0$. It follows that $v\left(Q_{i-1}\right) \leqslant v\left(D Q_{i-1}\right)=v\left(Q_{j-1}^{\prime}\right)+v(f)$. Moreover,

$$
\begin{aligned}
v\left(A_{j}^{\prime}\right) & \leqslant v\left(A_{j}^{\prime}\right)-v\left(Q_{i-1}\right)+v\left(Q_{j-1}^{\prime}\right)+v(f) \\
& =-v\left(Q_{i-1}\right)-v\left(f Q_{j}-1 S-g P_{j-1}^{\prime}\right)+v(f)+v(g) \\
& =-v\left(Q_{i-1}\right)-v\left(D Q_{i-1} S-D P_{i-1}\right)+v(f)+v(g) \\
& \leq-v\left(Q_{i-1}\right)-v\left(Q_{i-1} S-P_{i-1}\right)+v(f)+v(g)=v\left(A_{i}\right)+v(f)+v(g),
\end{aligned}
$$

where we used again [4, eq. (6)] in the last step. $\square$

Theorem 4. If $S$ has a good LCP and $f, g \in F[x]$ with $f g \neq 0$, then the sequence with generating function $\operatorname{Fr}\left(\frac{\mathrm{F}}{\mathrm{g}} \mathrm{S}\right)$ has a good LCP.

Proof. We use the notation in Lemma 3 and recall that $v(f)=\operatorname{deg}(f)$ for any $f \in F[x]$. We have $\operatorname{Fr}\left(\frac{E}{g} S\right)=\left[0, A_{1}, A_{2}^{\prime}, \ldots\right]$. The hypothesis and Theorem 3 yield $v\left(A_{i}\right) \leq C \log i$ for all $i \geq 1$. Now let $j \geqslant 1$ be such that $v\left(A_{j}^{\prime}\right)>v(f)+v(g)$. Then Lemma 3 implies $v\left(A_{j}^{\prime}\right) \leq C \log i+v(f)+v(g)$ and $i \leq v\left(Q_{i-1}\right)+1 \leq v\left(Q_{j-1}^{\prime}\right)+v(f)+1$, thus

$$
v\left(A_{j}^{\prime}\right) \leq C \log \left(v\left(Q_{j-1}^{\prime}\right)+v(f)+1\right)+v(f)+v(g) .
$$

This holds trivially if $v\left(A_{j}^{\prime}\right) \leq v(f)+v(g)$, and so $(G)$ holds for all $j \geqslant 1$. Now let $n \geqslant 1$ be arbitrary, then with a suitable $j \geqslant 0$ we get by Lemma 1 and ( 6 ),

$$
\begin{aligned}
& \left|L_{n}\left(F_{r}\left(\frac{f}{g} S\right)\right)-\frac{n}{2}\right| \leq \frac{1}{2} \max \left(v\left(A_{j}^{\prime}\right), v\left(A_{j+1}^{\prime}\right)\right) \\
& \quad \leq \frac{C}{2} \log \left(v\left(Q_{j}^{\prime}\right)+v(E)+1\right\rangle+\frac{1}{2} v(f)+\frac{1}{2} v(g) \\
& \quad \leq \frac{C}{2} \log (n+v(f)+1)+\frac{1}{2} v(f)+\frac{1}{2} v(g) \leq C_{1} \log n
\end{aligned}
$$

with a suitable constant $c_{1}$.

Corollary 1. If $S$ has a good LCP, then every shifted sequence $s_{m}, m=1,2, \ldots$, has a good LCP.

Proof. The generating function of $S_{m}$ is given by $F_{r}\left(x^{m} s\right)$, and so the desired result follows from Theorem 4. 

For $S \in G$ we put $K(S)=\begin{aligned} & s u p \\ & j \geq 1\end{aligned} \operatorname{deg}\left(A_{j}\right)$. If the sequence $S$ has an irra-

tional generating function and $\mathrm{K}(\mathrm{S})<\infty$, then Theorem 3 shows that $S$ has a good LCP. Thus by Corollary 1 , every shifted sequence $S_{m}$ has a good LCP. More precisely we have the following.

Theorem 5. Let $S \in G$ with $K(S)<\infty$ and $f, g \in F[x]$ with $f_{g} \neq 0$. Then

$$
K\left(\frac{f}{g} S\right) \leq K(S)+\operatorname{deg}(f)+\operatorname{deg}(g) .
$$

Proof. If in the notation of Lemma 3 we have $v\left(A_{j}^{\prime}\right)>v(E)+v(g)$ for some $j \geqslant 1$, then by this lemma we get $v\left(A_{j}^{\prime}\right) \leq K(S)+v(f)+v(g)$, and the latter inequality holds trivially if $v\left(A_{j}^{\prime}\right) \leq v(f)+v(g)$. Recall also that $v(f)=\operatorname{deg}(f)$ for any $f \in F[x]$.

Corollary 2. If $\mathrm{K}(\mathrm{S})<\infty$, then $\mathrm{K}\left(\mathrm{S}_{\mathrm{m}}\right) \leqslant \mathrm{K}(\mathrm{s})+\mathrm{m}$ for $\mathrm{m}=1,2, \ldots$.

Proof. This follows from Theorem 5 since the generating function of $S_{m}$ is $\operatorname{Fr}\left(x^{m} S\right)$.

In particular, Corollary 2 shows that if $K(S)<\infty$, then $K\left(S_{m}\right)<\infty$ for all $\mathrm{m}$. The result of Corollary 2 is in general best possible, as is proved by considering the following generalized Rueppel sequence $S$ constructed in [3]. Let $F=F_{2}$ and let $s_{1}=1$ if $1=2^{j}-1$ for some $j \geqslant 1$ and $s_{1}=0$ otherwise. Then $k(s)=1$ by $[3, p .232]$. Now let $m=2^{j}-1$ for some $j \geq 1$. Then the generating function of $s_{m}$ has the form $x^{-2^{j}}+$ smaller powers, and so the first partial quotient of $s_{m}$ has degree $2^{j}$. Thus $k\left(s_{m}\right) \geq 2^{j}$. On the other hand, corollary 2 yields $k\left(s_{m}\right) \leq$ $K(s)+m=2^{j}$, hence $K\left(S_{m}\right)=2^{j}$. Thus for this sequence $s$ we have $K\left(S_{m}\right)=K(S)+m$ for infinitely many $\mathrm{m}$.

\section{SEQUENCES WITH A UNIFORMLY GOOD LINEAR COMPLEXITY PROFILE}

It follows from Corollary 1 that if $S$ has a good LCP, then for every $m \geqslant 0$ there exists a constant $C_{m}$ (which may depend on $S$ and $m$ ) such that

$$
\left|L_{n}\left(s_{m}\right)-\frac{\pi}{2}\right| \leq c_{m} \text { Log } n \quad \text { for all } n \geq 1 \text {. }
$$

In this context it is of interest to consider the following notion.

Definition 2. A sequence $S$ of elements of the field $F$ has a uniformly good LCP if there exists a constant $C$ (which may depend on $S$ but not on $m$ ) such that 


$$
\left|L_{n}\left(s_{m}\right)-\frac{n}{2}\right| \leqslant C \log n \quad \text { for all } m \geqslant 0 \text { and } n \geq 1
$$

Theorem 6. If $F=F_{q}$, then the set of all sequences with a uniformly good LCP has h-measure 0 .

Proof. Let $W$ be the set in question. Let the sequence $S \in F_{q}^{\infty}$ be such that it contains somewhere a string of zeros of length $\tau \geqslant 1$, and let $s_{k+1}$ be the first term of this string. Then $L_{r}\left(S_{k}\right)=0$, hence if also $S \in W$, then it follows from Definition 2 that

$$
\left|L_{r}\left(s_{k}\right)-\frac{r}{2}\right|=\frac{r}{2} \leqslant \text { C Log } r .
$$

This implies that $r$ is bounded from above by a constant which depends only on $C$. Thus $W \subseteq B$, where $B$ is the set of all $S \in F_{q}^{\infty}$ for which all strings of zeros have bounded length. We have $B=\bigcup_{r=1}^{\infty} B_{r}$, where ${ }_{r}^{q}$ is the set of all $S \in F_{q}^{\infty}$ for which all strings of zeros have length $\leq r$. Fix $r \geqslant 1$, and for any integer $t \geq 0$ let $B_{r}^{(t)}$ be the set of all $S \in F_{q}^{\infty}$ with the following property: for each $j=0,1, \ldots, t$ at least one of the terms $s_{j(r+1)+1}, s_{j(r+1)+2} \cdots, s(j+1)(r+1)$ is $\neq 0$. Then $B_{r} \subseteq \bigcap_{t=0}^{\infty} B_{r}^{(t)}$. The prabability that at least one of any $r+1$ consecutive $s_{i}$ is $\neq 0$ is given by $1-q^{-r-1}$, and so $h\left(B_{r}^{(t)}\right)=\left(1-q^{-r-1}\right)^{t+1}$. It follows that

$$
h\left(B_{r}\right) \leq h\left(\bigcap_{t=0}^{\infty} B_{r}^{(t)}\right) \leq\left(1-q^{-r-1}\right)^{t+1} \quad \text { for all } t \geq 0 \text {, }
$$

and letting $t \rightarrow \infty$ on the right we get $h\left(B_{r}\right)=0$. This yields $h(B)=0$, and so $W \subseteq B$ implies $h(W)=0$.

Theorem 6 shows that having a uniformly good LCP is not a typical property of a random sequence of elements of $F_{q}$. Sequences with a uniformily good LCP can also be characterized in terms of continued fraction expansions. Let $\left[0, A_{1}^{(m)}, A_{2}^{(m)}, \ldots\right]$ be the continued fraction expansion of the generating function of the shifted sequence $s_{m}, m=0,1, \ldots$.

Theorem 7. A sequence $S$ has a uniformly good LCP if and only if its generating function is irrational and there exists a constant $C$ (which may depend on $S$ but not on $m$ ) such that $\operatorname{deg}\left(A_{j}^{(m)}\right) \leq C$ Log $j$ for all $j \geq 1$ and $m \geq 0$.

Proof. This follows from Propositions 1 and 2 .

It is an open question whether there actually exists a sequence with a uniformly good LCP. Another open question is to decide whether there exists a sequence $S$ for 
which $s \quad u \quad p \quad K\left(S_{m}\right)<\infty$. A positive answer to the second question will of course imply a positive answer to the first question.

\section{REFERENCES}

[1] U. Krengel: Ergodic Theorems, de Gruyter, Berlin, 1985.

[2] M. Loève: Probability Theory, 3rd ed., Van Nostrand, New York, 1963.

[3] H. Niederreiter: Continued fractions for formal power series, pseudorandom numbers, and linear complexity of sequences, Contributions to General Algebra 5 (Proc. Salzburg Conf., 1986), Pp. 221-233, Teubner, Stuttgart, 1987.

[4] H. Niederreiter: Sequences with almost perfect linear complexity profile, Advances in Cryptology - EURoCRyPT 187 (D. Chaum and w. L. Price, eds.), Lecture Notes in Computer Science, Vol. 304, pp. 37-51, Springer, Berlin, 1988.

[5] H. Niederreiter: The probabilistic theory of linear complexity, Advances in Cryptology - EUROCRYPT 188 (C. G. Günther, ed.), Lecture Notes in Computer Science, Val. 330, pp. 191-209, Springer, Berlin, 1988.

[6] F. Piper: Stream ciphers, Elektrotechnik und Maschinenbau 104, 564-568 (1987).

[7] R. A. Rueppel: Linear complexity and random sequences, Advances in Cryptology EUROCRYPT '85 (F. Pichler, ed.), Lecture Notes in Computer Science, Vol. 219, pp. 167-188, Springer, Berlin, 1986.

[8] R. A. Rueppel: Analysis and Design of Stream Ciphers, Springer, Berlin, 1986. 